\title{
Research Article \\ Existence of Positive, Negative, and Sign-Changing Solutions to Discrete Boundary Value Problems
}

\author{
Bo Zheng, ${ }^{1}$ Huafeng Xiao, ${ }^{1}$ and Haiping Shi ${ }^{2}$ \\ ${ }^{1}$ School of Mathematics and Information Sciences, Guangzhou University, Guangzhou, \\ Guangdong 510006, China \\ ${ }^{2}$ Department of Basic Courses, Guangdong Baiyun Institute, Guangzhou, Guangdong 510450, China
}

Correspondence should be addressed to Bo Zheng, zhengbo611@yahoo.com.cn

Received 11 November 2010; Accepted 15 February 2011

Academic Editor: Zhitao Zhang

Copyright (C) 2011 Bo Zheng et al. This is an open access article distributed under the Creative Commons Attribution License, which permits unrestricted use, distribution, and reproduction in any medium, provided the original work is properly cited.

By using critical point theory, Lyapunov-Schmidt reduction method, and characterization of the Brouwer degree of critical points, sufficient conditions to guarantee the existence of five or six solutions together with their sign properties to discrete second-order two-point boundary value problem are obtained. An example is also given to demonstrate our main result.

\section{Introduction}

Let $\mathbb{N}, \mathbb{Z}$, and $\mathbb{R}$ denote the sets of all natural numbers, integers, and real numbers, respectively. For $a, b \in \mathbb{Z}$, define $\mathbb{Z}(a, b)=\{a, a+1, \ldots, b\}$, when $a \leq b$. $\Delta$ is the forward difference operator defined by $\Delta u(n)=u(n+1)-u(n), \Delta^{2} u(n)=\Delta(\Delta u(n))$.

Consider the following discrete second-order two-point boundary value problem (BVP for short):

$$
\begin{gathered}
\Delta^{2} u(n-1)+V^{\prime}(u(n))=0, \quad n \in \mathbb{Z}(1, T), \\
u(0)=0=u(T+1),
\end{gathered}
$$

where $V \in C^{2}(\mathbb{R}, \mathbb{R}), T \geq 1$ is a given integer.

By a solution $u$ to the $\operatorname{BVP}(1.1)$, we mean a real sequence $\{u(n)\}_{n=0}^{T+1}(=(u(0)$, $u(1), \ldots, u(T+1)))$ satisfying (1.1). For $u=\{u(n)\}_{n=0}^{T+1}$ with $u(0)=0=u(T+1)$, we say that $u \neq 0$ if there exists at least one $n \in \mathbb{Z}(1, T)$ such that $u(n) \neq 0$. We say that $u$ is positive (and write $u>0$ ) if for all $n \in \mathbb{Z}(1, T), u(n) \geq 0$, and $\{n \in \mathbb{Z}(1, T): u(n)>0\} \neq \emptyset$, and similarly, 
$u$ is negative $(u<0)$ if for all $n \in \mathbb{Z}(1, T), u(n) \leq 0$, and $\{n \in \mathbb{Z}(1, T): u(n)<0\} \neq \emptyset$. We say that $u$ is sign-changing if $u$ is neither positive nor negative. Under convenient assumptions, we will prove the existence of five or six solutions to (1.1), which include positive, negative, and sign-changing solutions.

Difference BVP has widely occurred as the mathematical models describing real-life situations in mathematical physics, finite elasticity, combinatorial analysis, and so forth; for example, see [1,2]. And many scholars have investigated difference BVP independently mainly for two reasons. The first one is that the behavior of discrete systems is sometimes sharply different from the behavior of the corresponding continuous systems. For example, every solution of logistic equation $x^{\prime}(t)=a x(t)(1-x(t) / k)$ is monotone, but its discrete analogue $\Delta x(n)=a x(n)(1-x(n) / k)$ has chaotic solutions; see [3] for details. The second one is that there is a fundamental relationship between solutions to continuous systems and the corresponding discrete systems by employing discrete variable methods [1]. The classical results on difference BVP employs numerical analysis and features from the linear and nonlinear operator theory, such as fixed point theorems. We remark that, usually, the application of the fixed point theorems yields existence results only.

Recently, however, a few scholars have used critical point theory to deal with the existence of multiple solutions to difference BVP. For example, in 2004, Agarwal et al. [4] employed the mountain pass lemma to study (1.1) with $V^{\prime}(u(n))=f(n, u(n))$ and obtained the existence of multiple solutions. Very recently, Zheng and Zhang [5] obtained the existence of exactly three solutions to (1.1) by making use of three-critical-point theorem and analytic techniques. We also refer to [6-9] for more results on the difference BVP by using critical point theory. The application of critical point theory to difference BVP represents an important advance as it allows to prove multiplicity results as well.

Here, by using critical point theory again, as well as Lyapunov-Schmidt reduction method and degree theory, a sharp condition to guarantee the existence of five or six solutions together with their sign properties to (1.1) is obtained. And this paper offers, to the best of our knowledge, a new method to deal with the sign of solutions in the discrete case.

Here, we assume that $V^{\prime}(0)=0$ and

$$
V^{\prime \prime}(\infty)=\lim _{|t| \rightarrow \infty} \frac{V^{\prime}(t)}{t} \in \mathbb{R}
$$

Hence, $V^{\prime}$ grows asymptotically linear at infinity.

The solvability of (1.1) depends on the properties of $V^{\prime}$ both at zero and at infinity. If

$$
V^{\prime \prime}(\infty)=\lambda_{l}, \quad\left(\text { or } V^{\prime \prime}(0)=\lim _{|t| \rightarrow 0} \frac{V^{\prime}(t)}{t}=\lambda_{l}\right),
$$

where $\lambda_{l}$ is one of the eigenvalues of the eigenvalue problem

$$
\begin{gathered}
\Delta^{2} u(n-1)+\lambda u(n)=0, \quad n \in \mathbb{Z}(1, T), \\
u(0)=0=u(T+1),
\end{gathered}
$$


then we say that (1.1) is resonant at infinity (or at zero); otherwise, we say that (1.1) is nonresonant at infinity (or at zero). On the eigenvalue problem (1.4), the following results hold (see [1] for details).

Proposition 1.1. For the eigenvalue problem (1.4), the eigenvalues are

$$
\lambda=\lambda_{l}=4 \sin ^{2} \frac{l \pi}{2(T+1)}, \quad l=1,2, \ldots, T,
$$

and the corresponding eigenfunctions with $\lambda_{l}$ are $\phi_{l}(n)=\sin (l \pi n /(T+1)), l=1,2, \ldots, T$.

Remark 1.2. (i) The set of functions $\left\{\phi_{l}(n), l=1,2, \ldots, T\right\}$ is orthogonal on $\mathbb{Z}(1, T)$ with respect to the weight function $r(n) \equiv 1$; that is,

$$
\sum_{n=1}^{T}\left(\phi_{l}(n), \phi_{j}(n)\right)=0, \quad \forall l \neq j .
$$

Moreover, for each $l \in \mathbb{Z}(1, T), \sum_{n=1}^{T} \sin ^{2}(l \pi n /(T+1))=(T+1) / 2$.

(ii) It is easy to see that $\phi_{l}$ is positive and $\phi_{l}$ changes sign for each $l \in \mathbb{Z}(2, T)$; that is, $\left\{n: \phi_{l}(n)>0\right\} \neq \emptyset$ and $\left\{n: \phi_{l}(n)<0\right\} \neq \emptyset$ for $l \in \mathbb{Z}(2, T)$.

The main result of this paper is as follows.

Theorem 1.3. If $V^{\prime \prime}(0)<\lambda_{1}, V^{\prime \prime}(\infty) \in\left(\lambda_{k}, \lambda_{k+1}\right)$ with $k \in \mathbb{Z}(2, T-1)$, and $0<V^{\prime \prime}(t) \leq \gamma<\lambda_{k+1}$, then (1.1) has at least five solutions. Moreover, one of the following cases occurs:

(i) $k$ is even and (1.1) has two sign-changing solutions,

(ii) $k$ is even and (1.1) has six solutions, three of which are of the same sign,

(iii) $k$ is odd and (1.1) has two sigh-changing solutions,

(iv) $k$ is odd and (1.1) has three solutions of the same sign.

Remark 1.4. The assumption $V^{\prime \prime}(0)<\lambda_{1}$ in Theorem 1.3 is sharp in the sense that when $\lambda_{k-1}<V^{\prime \prime}(0)<\lambda_{k}, \lambda_{k}<V^{\prime \prime}(\infty)<\lambda_{k+1}$ for $k \in \mathbb{Z}(2, T-1)$, Theorem 1.4 of [5] gives sufficient conditions for (1.1) to have exactly three solutions with some restrictive conditions.

Example 1.5. Consider the BVP

$$
\begin{gathered}
\Delta^{2} u(n-1)+V^{\prime}(u(n))=0, \quad n \in \mathbb{Z}(1,5), \\
u(0)=0=u(6),
\end{gathered}
$$


where $V \in C^{2}(\mathbb{R}, \mathbb{R})$ is defined as follows:

$$
V^{\prime}(t)= \begin{cases}\arctan t-\frac{4 t}{5}, & |t| \leq \frac{1}{3}, \\ \text { a strictly increasing function satisfying } \frac{1}{10} \leq V^{\prime \prime}(t) \leq \frac{49}{20}, & \frac{1}{3} \leq|t| \leq 1, \\ \frac{(\arctan t)}{10}+\frac{12 t}{5}, & |t| \geq 1 .\end{cases}
$$

It is easy to verify that $V^{\prime}(0)=0, V^{\prime \prime}(0)=1 / 5<\lambda_{1}=2-\sqrt{3}, V^{\prime \prime}(\infty)=12 / 5 \in(2,3)=\left(\lambda_{3}, \lambda_{4}\right)$, and $0<V^{\prime \prime}(t) \leq 49 / 20<3=\lambda_{4}$. So, all the conditions in Theorem 1.3 are satisfied with $k=3$. And hence (1.7) has at least five solutions, among which two sign-changing solutions or three solutions of the same sign.

By the computation of critical groups, for $k=1$, we have the following.

Corollary 1.6 (see Remark 3.7 below). If $V^{\prime \prime}(0)<\lambda_{1}, V^{\prime \prime}(\infty) \in\left(\lambda_{1}, \lambda_{2}\right)$, and $0<V^{\prime \prime}(t) \leq \gamma<\lambda_{2}$, then (1.1) has at least one positive solution and one negative solution.

\section{Preliminaries}

Let

$$
E=\{u: \mathbb{Z}(0, T+1) \longrightarrow \mathbb{R}, u(0)=0=u(T+1)\} .
$$

Then, $E$ is a $T$-dimensional Hilbert space with inner product

$$
(u, v)=\sum_{n=0}^{T}(\Delta u(n), \Delta v(n)), \quad u, v \in E,
$$

by which the norm $\|\cdot\|$ can be induced by

$$
\|u\|=\left(\sum_{n=0}^{T}|\Delta u(n)|^{2}\right)^{1 / 2}, \quad u \in E .
$$

Here, $|\cdot|$ denotes the Euclidean norm in $\mathbb{R}$, and $(\cdot, \cdot)$ denotes the usual inner product in $\mathbb{R}$. Define

$$
J(u)=\frac{1}{2} \sum_{n=0}^{T}|\Delta u(n)|^{2}-\sum_{n=1}^{T} V(u(n)), \quad u \in E .
$$


Then, the functional $J$ is of class $C^{2}$ with

$$
\begin{aligned}
\left\langle J^{\prime}(u), v\right\rangle & =\sum_{n=0}^{T}(\Delta u(n), \Delta v(n))-\sum_{n=1}^{T}\left(V^{\prime}(u(n)), v(n)\right) \\
& =-\sum_{n=1}^{T}\left(\Delta^{2} u(n-1)+V^{\prime}(u(n)), v(n)\right), \quad u, v \in E .
\end{aligned}
$$

So, solutions to (1.1) are precisely the critical points of $J$ in $E$.

As we have mentioned, we will use critical point theory, Lyapunov-Schmidt reduction method, and degree theory to prove our result. Let us collect some results that will be used below. One can refer to [10-12] for more details.

Let $E$ be a Hilbert space and $J \in C^{1}(E, \mathbb{R})$. Denote

$$
J^{c}=\{u \in E: J(u) \leq c\}, \quad \mathcal{K}=\left\{u \in E: J^{\prime}(u)=0\right\}, \quad \mathcal{K}_{c}=\{u \in \mathcal{K}: J(u)=c\},
$$

for $c \in \mathbb{R}$. The following is the definition of the Palais-Smale (PS) compactness condition.

Definition 2.1. The functional $J$ satisfies the (PS) condition if any sequence $\left\{u_{m}\right\} \subset E$ such that $J\left(u_{m}\right)$ is bounded and $J^{\prime}\left(u_{m}\right) \rightarrow 0$ as $m \rightarrow \infty$ has a convergent subsequence.

In [13], Cerami introduced a weak version of the (PS) condition as follows.

Definition 2.2. The functional $J$ satisfies the Cerami (C) condition if any sequence $\left\{u_{m}\right\} \subset E$ such that $J\left(u_{m}\right)$ is bounded and $\left(1+\left\|u_{m}\right\|\right)\left\|J^{\prime}\left(u_{m}\right)\right\| \rightarrow 0$, as $m \rightarrow \infty$ has a convergent subsequence.

If $J$ satisfies the (PS) condition or the (C) condition, then $J$ satisfies the following deformation condition which is essential in critical point theory (cf. $[14,15])$.

Definition 2.3. The functional $J$ satisfies the $\left(\mathrm{D}_{c}\right)$ condition at the level $c \in \mathbb{R}$ if for any $\bar{\epsilon}>0$ and any neighborhood $\mathcal{N}$ of $\mathcal{K}_{c}$, there are $\epsilon>0$ and a continuous deformation $\eta:[0,1] \times E \rightarrow$ E such that

(i) $\eta(0, u)=u$ for all $u \in E$,

(ii) $\eta(t, u)=u$ for all $u \notin J^{-1}([c-\bar{\epsilon}, c+\bar{\epsilon}])$,

(iii) $J(\eta(t, u))$ is non-increasing in $t$ for any $u \in E$,

(iv) $\eta\left(1, J^{c+\epsilon} \backslash \mathcal{N}\right) \subset J^{c-\epsilon}$.

$J$ satisfies the $(\mathrm{D})$ condition if $J$ satisfies the $\left(\mathrm{D}_{c}\right)$ condition for all $c \in \mathbb{R}$.

Let $H_{*}$ denote singular homology with coefficients in a field $\mathbb{F}$. If $u \in E$ is a critical point of $J$ with critical level $c=J(u)$, then the critical groups of $u$ are defined by

$$
C_{q}(J, u)=H_{q}\left(J^{c}, J^{c} \backslash\{u\}\right), \quad q \in \mathbb{Z}(0) .
$$


Suppose that $J(\mathcal{K})$ is strictly bounded from below by $a \in \mathbb{R}$ and that $J$ satisfies $\left(\mathrm{D}_{c}\right)$ for all $c \leq a$. Then, the $q$ th critical group at infinity of $J$ is defined in [16] as

$$
C_{q}(J, \infty)=H_{q}\left(E, J^{a}\right), \quad q \in \mathbb{Z}(0) .
$$

Due to the condition $\left(D_{c}\right)$, these groups are not dependent on the choice of $a$.

Assume that $\# \mathcal{K}<\infty$ and $J$ satisfies the (D) condition. The Morse-type numbers of the pair $\left(E, J^{a}\right)$ are defined by $M_{q}=M_{q}\left(E, J^{a}\right)=\sum_{u \in \mathcal{K}} \operatorname{dim} C_{q}(J, u)$, and the Betti numbers of the pair $\left(E, J^{a}\right)$ are defined by $\beta_{q}=\operatorname{dim} C_{q}(J, \infty)$. By Morse theory [10,11], the following relations hold:

$$
\begin{gathered}
\sum_{j=0}^{q}(-1)^{q-j} M_{j} \geq \sum_{j=0}^{q}(-1)^{q-j} \beta_{j}, \quad q \in \mathbb{Z}(0), \\
\sum_{q=0}^{\infty}(-1)^{q} M_{q}=\sum_{q=0}^{\infty}(-1)^{q} \beta_{q} .
\end{gathered}
$$

It follows that $M_{q} \geq \beta_{q}$ for all $q \in \mathbb{Z}(0)$. If $\mathcal{K}=\emptyset$, then $\beta_{q}=0$ for all $q \in \mathbb{Z}(0)$. Thus, when $\beta_{q} \neq 0$ for some $q \in \mathbb{Z}(0)$, $J$ must have a critical point $u$ with $C_{q}(J, u) \supsetneqq 0$.

The critical groups of $J$ at an isolated critical point $u$ describe the local behavior of $J$ near $u$, while the critical groups of $J$ at infinity describe the global property of $J$. In most applications, unknown critical points will be found from (2.9) or (2.10) if we can compute both the critical groups at known critical points and the critical groups at infinity. Thus, the computation of the critical groups is very important. Now, we collect some useful results on computation of critical groups which will be employed in our discussion.

Proposition 2.4 (see [16]). Let $E$ be a real Hilbert space and $J \in C^{1}(E, \mathbb{R})$. Suppose that $E$ splits as $E=X \oplus Y$ such that $J$ is bounded from below on $Y$ and $J(x) \rightarrow-\infty$ for $x \in X$ as $\|x\| \rightarrow \infty$. Then $C_{k}(J, \infty) \neq 0$ for $k=\operatorname{dim} X<\infty$.

Proposition 2.5 (see [17]). Let E be a separable Hilbert space with inner product $\langle\cdot, \cdot\rangle$ and corresponding norm $\|\cdot\|, X, Y$ closed subspaces of $E$ such that $E=X \oplus Y$. Assume that $J \in C^{1}(E, \mathbb{R})$ satisfies the (PS) condition and the critical values of $J$ are bounded from below. If there is a real number $m>0$ such that for all $v \in X$ and $w_{1}, w_{2} \in Y$, there holds

$$
\left\langle\nabla J\left(v+w_{1}\right)-\nabla J\left(v+w_{2}\right), w_{1}-w_{2}\right\rangle \geq m\left\|w_{1}-w_{2}\right\|^{2},
$$

then there exists a $C^{1}$-functional $\varphi: X \rightarrow \mathbb{R}$ such that

$$
C_{q}(J, \infty) \cong C_{q}(\varphi, \infty), \quad q \in \mathbb{Z}(0) .
$$

Moreover, if $k=\operatorname{dim} X<\infty$ and $C_{k}(J, \infty) \neq 0$, then $C_{q}(J, \infty) \cong \delta_{q, k} \mathbb{F}$.

Let $B_{r}$ denote the open ball in $E$ about 0 of the radius $r$, and let $\partial B_{r}$ denote its boundary.

Lemma 2.6 (Mountain Pass Lemma $[10,11]$ ). Let $E$ be a real Banach space and $J \in C^{1}(E, \mathbb{R})$ satisfying the (PS) condition. Suppose that $J(0)=0$ and 
(J1) there are constants $\rho>0, a>0$ such that $\left.J\right|_{\partial B_{\rho}} \geq a>0$, and

(J2) there is a $u_{0} \in E \backslash B_{\rho}$ such that $J\left(u_{0}\right) \leq 0$.

Then, J possesses a critical value $c \geq a$. Moreover, $c$ can be characterized as

$$
c=\inf _{h \in \Gamma} \sup _{s \in[0,1]} J(h(s))
$$

where

$$
\Gamma=\left\{h \in C([0,1], E) h(0)=0, h(1)=u_{0}\right\} .
$$

Definition 2.7 (Mountain pass point). An isolated critical point $u$ of $J$ is called a mountain pass point if $C_{1}(J, u) \nsubseteq 0$.

To compute the critical groups of a mountain pass point, we have the following result.

Proposition 2.8 (see [11]). Let E be a real Hilbert space. Suppose that $J \in C^{2}(E, \mathbb{R})$ has a mountain pass point $u$ and that $J^{\prime \prime}(u)$ is a Fredholm operator with finite Morse index satisfying

$$
J^{\prime \prime}(u) \geq 0, \quad 0 \in \sigma\left(J^{\prime \prime}(u)\right) \Longrightarrow \operatorname{dim} \operatorname{ker}\left(J^{\prime \prime}(u)\right)=1 .
$$

Then,

$$
C_{q}(J, u) \cong \delta_{q, 1} \mathbb{F}, \quad q \in \mathbb{Z}(0) .
$$

The following theorem gives a relation between the Leray-Schauder degree and the critical groups.

Theorem 2.9 (see $[10,11]$ ). Let $E$ be a real Hilbert space, and let $J \in C^{2}(E, \mathbb{R})$ be a function satisfying the (PS) condition. Assume that $J^{\prime}(x)=x-A x$, where $A: E \rightarrow E$ is a completely continuous operator. If $u$ is an isolated critical point of $J$, that is, there exists a neighborhood $U$ of $u$, such that $u$ is the only critical point of $J$ in $\bar{U}$, then

$$
d(I-A, U, 0)=\sum_{q=0}^{\infty}(-1)^{q} \operatorname{dim} C_{q}(J, u)
$$

where d denotes the Leray-Schauder degree.

Finally, we state a global version of the Lyapunov-Schmidt reduction method.

Lemma 2.10 (see [18]). Let $E$ be a real separable Hilbert space. Let $X$ and $Y$ be closed subspaces of $E$ such that $E=X \oplus Y$ and $J \in C^{1}(E, \mathbb{R})$. If there are $m>0, \alpha>1$ such that for all $x \in X, y, y_{1} \in Y$,

$$
\left\langle J^{\prime}(x+y)-J^{\prime}\left(x+y_{1}\right), y-y_{1}\right\rangle \geq m\left\|y-y_{1}\right\|^{\alpha},
$$

then the following results hold. 
(i) There exists a continuous function $\psi: X \rightarrow Y$ such that

$$
J(x+\psi(x))=\min _{y \in Y} J(x+y)
$$

Moreover, $\psi(x)$ is the unique member of $Y$ such that

$$
\left\langle J^{\prime}(x+\psi(x)), y\right\rangle=0, \quad \forall y \in Y .
$$

(ii) The function $\bar{J}: X \rightarrow \mathbb{R}$ defined by $\bar{J}(x)=J(x+\psi(x))$ is of class $C^{1}$, and

$$
\left\langle\bar{J}^{\prime}(x), x_{1}\right\rangle=\left\langle J^{\prime}(x+\psi(x)), x_{1}\right\rangle, \quad \forall x, x_{1} \in X
$$

(iii) An element $x \in X$ is a critical point of $\bar{J}$ if and only if $x+\psi(x)$ is a critical point of J.

(iv) Let $\operatorname{dim} X<\infty$ and $P$ be the projection onto $X$ across $Y$. Let $S \subset X$ and $\Sigma \subset E$ be open bounded regions such that

$$
\{x+\psi(x): x \in S\}=\Sigma \cap\{x+\psi(x): x \in X\} .
$$

If $\bar{J}^{\prime}(x) \neq 0$ for $x \in \partial S$, then

$$
d\left(\bar{J}^{\prime}, S, 0\right)=d\left(J^{\prime}, \Sigma, 0\right)
$$

where d denotes the Leray-Schauder degree.

(v) If $u=x+\psi(x)$ is a critical point of mountain pass type of $J$, then $x$ is a critical point of mountain pass type of $\bar{J}$.

\section{Proof of Theorem 1.3}

In this section, firstly, we obtain a positive solution $u^{+}$and a negative solution $u^{-}$with $C_{q}\left(J, u^{+}\right) \cong C_{q}\left(J, u^{-}\right) \cong \delta_{q, 1} \mathbb{F}$ to $(1.1)$ by using cutoff technique and the mountain pass lemma. Then, we give a precise computation of $C_{q}(J, 0)$. And we remark that under the assumptions of Theorem 1.3, $C_{q}(J, \infty)$ can be completely computed by using Propositions 2.4 and 2.5. Based on these results, four nontrivial solutions $\left\{u^{+}, u^{-}, u_{0}, u_{1}\right\}$ to (1.1) can be obtained by (2.9) or (2.10). However, it seems difficult to obtain the sign property of $u_{0}$ and $u_{1}$ through their depiction of critical groups. To conquer this difficulty, we compute the Brouwer degree of the sets of positive solutions and negative solutions to (1.1). Finally, the third nontrivial solution to (1.1) is obtained by Lyapunov-Schmidt reduction method, and its characterization of the local degree results in one or two more nontrivial solutions to (1.1) together with their sign property. 
Let

$$
V^{\prime+}(t)=\left\{\begin{array}{ll}
V^{\prime}(t), & t \geq 0, \\
V^{\prime \prime}(0) t, & t<0,
\end{array} \quad V^{\prime-}(t)= \begin{cases}V^{\prime}(t), & t \leq 0 \\
V^{\prime \prime}(0) t, & t>0\end{cases}\right.
$$

and $V^{ \pm}(x)=\int_{0}^{x} V^{\prime \pm}(s) d s$. The functionals $J^{ \pm}: E \rightarrow \mathbb{R}$ are defined as

$$
J^{ \pm}(u)=\frac{1}{2}\|u\|^{2}-\sum_{n=1}^{T} V^{ \pm}(u(n))
$$

Remark 3.1. From the definitions of $V^{ \pm}$and $V^{\prime \prime}(0)<\lambda_{1}$, it is easy to see that if $u \in E$ is a critical point of $J^{+}$(or $J^{-}$), then $u>0$ (or $u<0$ ).

Lemma 3.2. The functionals $J^{ \pm}$satisfy the (PS) condition; that is, every sequence $\left\{u_{m}\right\}$ in $E$ such that $J^{ \pm}\left(u_{m}\right)$ is bounded, and $J^{ \pm}\left(u_{m}\right) \rightarrow 0$ as $m \rightarrow \infty$ has a convergent subsequence.

Proof. We only prove the case of $J^{+}$. The case of $J^{-}$is completely similar. Since $E$ is finite dimensional, it suffices to show that $\left\{u_{m}\right\}$ is bounded. Suppose that $\left\{u_{m}\right\}$ is unbounded. Passing to a subsequence, we may assume that $\left\|u_{m}\right\| \rightarrow \infty$ and for each $n$, either $\left|u_{m}(n)\right| \rightarrow$ $\infty$ or $\left\{u_{m}(n)\right\}$ is bounded.

Set $w_{m}=u_{m} /\left\|u_{m}\right\| \in E$. For a subsequence, $w_{m}$ converges to some $w$ with $\|w\|=1$. Since for all $\varphi \in E$, we have

$$
\left\langle J^{\prime+}\left(u_{m}\right), \varphi\right\rangle=\sum_{n=0}^{T}\left(\Delta u_{m}(n), \Delta \varphi(n)\right)-\sum_{n=1}^{T}\left(V^{\prime+}\left(u_{m}(n)\right), \varphi(n)\right)
$$

Hence,

$$
\frac{\left\langle J^{\prime+}\left(u_{m}\right), \varphi\right\rangle}{\left\|u_{m}\right\|}=\sum_{n=0}^{T}\left(\Delta w_{m}(n), \Delta \varphi(n)\right)-\sum_{n=1}^{T}\left(\frac{V^{\prime+}\left(u_{m}(n)\right)}{\left\|u_{m}\right\|}, \varphi(n)\right)
$$

If $\left|u_{m}(n)\right| \rightarrow \infty$, then

$$
\lim _{m \rightarrow \infty} \frac{V^{\prime+}\left(u_{m}(n)\right)}{\left\|u_{m}\right\|}=\lim _{m \rightarrow \infty} \frac{V^{\prime+}\left(u_{m}(n)\right)}{u_{m}(n)} w_{m}(n)=V^{\prime \prime}(\infty) w^{+}(n)+V^{\prime \prime}(0) w^{-}(n),
$$

where $w^{+}(n)=\max \{w(n), 0\}, w^{-}(n)=\min \{w(n), 0\}$. If $\left\{u_{m}(n)\right\}$ is bounded, then

$$
\lim _{m \rightarrow \infty} \frac{V^{\prime+}\left(u_{m}(n)\right)}{\left\|u_{m}\right\|}=0, \quad w(n)=0 .
$$


Letting $m \rightarrow \infty$ in (3.4), we have

$$
\sum_{n=0}^{T}(\Delta w(n), \Delta \varphi(n))-\sum_{n=1}^{T}\left(V^{\prime \prime}(\infty) w^{+}(n)+V^{\prime \prime}(0) w^{-}(n), \varphi(n)\right)=0
$$

which implies that $w(n)$ satisfies

$$
\begin{gathered}
\Delta^{2} w(n-1)+V^{\prime \prime}(\infty) w^{+}(n)+V^{\prime \prime}(0) w^{-}(n)=0, \quad n \in \mathbb{Z}(1, T) \\
w(0)=0=w(T+1) .
\end{gathered}
$$

Because $V^{\prime \prime}(0)<\lambda_{1}$, we see that if $w \neq 0$ is a solution to (3.8), then $u$ is positive. Since this contradicts $V^{\prime \prime}(\infty) \in\left(\lambda_{k}, \lambda_{k+1}\right)$, we conclude that $w \equiv 0$ is the only solution to (3.8). A contradiction to $\|w\|=1$.

Lemma 3.3. Under the conditions of Theorem 1.3, $J^{+}$has a positive mountain pass-type critical point $u^{+}$with $C_{q}\left(J^{+}, u^{+}\right) \cong C_{q}\left(J, u^{+}\right) \cong \delta_{q, 1} \mathbb{F} ; J^{-}$has a negative mountain pass-type critical point $u^{-}$with $C_{q}\left(J^{-}, u^{-}\right) \cong C_{q}\left(J, u^{-}\right) \cong \delta_{q, 1} \mathbb{F}$.

Proof. We only prove the case of $J^{+}$. Firstly, we will prove that $J^{+}$satisfies all the conditions in Lemma 2.6. And hence, $J^{+}$has at least one nonzero critical point $u^{+}$. In fact, $J^{+} \in C^{1}(E, \mathbb{R})$, and $J^{+}$satisfies the (PS) condition by Lemma 3.2. Clearly, $J^{+}(0)=0$. Thus, we still have to show that $J^{+}$satisfies (J1), (J2). To verify (J1), set $\alpha:=V^{\prime \prime}(0)<\lambda_{1}$, then for any $\epsilon>0$, there exists $\rho_{1}>0$, such that

$$
V^{\prime \prime}(t) \leq V^{\prime \prime}(0)+\epsilon=\alpha+\epsilon, \quad \text { for }|t| \leq \rho_{1} .
$$

So, by Taylor series expansion,

$$
V(t) \leq \frac{1}{2}(\alpha+\epsilon) t^{2}, \quad \text { for }|t| \leq \rho_{1}
$$

Take $\epsilon=\left(\lambda_{1}-\alpha\right) / 2>0$, then $\alpha+\epsilon=\left(\lambda_{1}+\alpha\right) / 2 \in\left(\alpha, \lambda_{1}\right)$. If we set $\rho_{2}=\left(\lambda_{1}+\alpha\right) / 2$, then

$$
V(t) \leq \frac{1}{2} \rho_{2} t^{2}, \quad \text { for }|t| \leq \rho_{1}
$$


Since for all $u \in E$, if $\|u\| \leq \sqrt{\lambda_{1}} \rho_{1}$, then $|u(n)| \leq \rho_{1}$ for every $n \in \mathbb{Z}(1, T)$ and hence

$$
\begin{aligned}
J^{+}(u) & =\frac{1}{2}\|u\|^{2}-\sum_{n=1}^{T} V^{+}(u(n)) \\
& =\frac{1}{2}\|u\|^{2}-\sum_{n \in N_{1}} V(u(n))-\frac{1}{2} \sum_{n \in N_{2}} \alpha(u(n), u(n)) \\
& \geq \frac{1}{2}\|u\|^{2}-\frac{1}{2} \rho_{2} \sum_{n \in N_{1}}(u(n), u(n))-\frac{1}{2} \alpha \sum_{n \in N_{2}}(u(n), u(n)) \\
& \geq \frac{1}{2}\|u\|^{2}-\frac{1}{2} \rho_{2} \sum_{n=1}^{T}(u(n), u(n)) \\
& \geq \frac{1}{2}\|u\|^{2}-\frac{1}{2} \frac{\rho_{2}}{\lambda_{1}}\|u\|^{2},
\end{aligned}
$$

where $N_{1}=\{n \in \mathbb{Z}(1, T) \mid u(n) \geq 0\}, N_{2}=\{n \in \mathbb{Z}(1, T) \mid u(n)<0\}$. If we take

$$
\rho=\sqrt{\lambda_{1}} \rho_{1}, \quad a=\frac{1}{2}\left(1-\frac{\rho_{2}}{\lambda_{1}}\right) \rho^{2}
$$

then $\left.J^{+}(u)\right|_{\partial B_{\rho}} \geq a>0$. And hence, (J1) holds.

To verify (J2), note that $V^{\prime \prime}(\infty) \in\left(\lambda_{k}, \lambda_{k+1}\right)$ implies that there exist $\bar{\gamma}>\lambda_{k}\left(>\lambda_{1}\right)$ and $b \in \mathbb{R}$, such that

$$
V(t) \geq \frac{\bar{\gamma}}{2} t^{2}+b, \quad \text { for } t \in \mathbb{R}
$$

So, if we take $\phi_{1}(n)>0$ with $\left\|\phi_{1}\right\|=1$, then

$$
\begin{aligned}
J^{+}\left(t \phi_{1}\right) & =\frac{t^{2}}{2}-\sum_{n=1}^{T} V\left(t \phi_{1}(n)\right) \\
& \leq \frac{t^{2}}{2}-\frac{\bar{\gamma} t^{2}}{2}\left(\phi_{1}, \phi_{1}\right)-b T \\
& =\frac{t^{2}}{2}-\frac{\bar{\gamma} t^{2}}{2 \lambda_{1}}-b T \longrightarrow-\infty, \quad 0<t \longrightarrow+\infty
\end{aligned}
$$

So, if we take $t$ sufficiently large such that $t>\rho$ and for $u_{0}=t e \in E, J^{+}\left(u_{0}\right) \leq 0$, then (J2) holds.

Now, by Lemma 2.6, $J^{+}$has at least a nonzero critical point $u^{+}$. And for all $n \in \mathbb{Z}(1, T)$, we claim that $u^{+}(n) \geq 0$. If not, set $A_{1}=\left\{n \in \mathbb{Z}(1, T) \mid u^{+}(n)<0\right\}$, then for all $n \in A_{1}, \Delta^{2} u^{+}(n-$ $1)+V^{\prime \prime}(0) u^{+}(n)=0$. By $V^{\prime \prime}(0)<\lambda_{1}, u^{+}(n) \equiv 0$ for all $n \in A_{1}$. Hence, $A_{1}=\emptyset$.

In the following, we will compute the critical groups $C_{q}\left(J^{+}, u^{+}\right)$by using Proposition 2.8. 
Assume that

$$
\left\langle J^{\prime \prime}\left(u^{+}\right) v, v\right\rangle=\langle v, v\rangle-\sum_{n=1}^{T}\left(V^{\prime \prime}\left(u^{+}(n)\right) v(n), v(n)\right) \geq 0, \quad \forall v \in E
$$

and that there exists $v_{0} \not \equiv 0$ such that

$$
\left\langle J^{\prime \prime}\left(u^{+}\right) v_{0}, v\right\rangle=0, \quad \forall v \in E .
$$

This implies that $v_{0}$ satisfies

$$
\begin{gathered}
\Delta^{2} v_{0}(n-1)+V^{\prime \prime}\left(u^{+}(n)\right) v_{0}(n)=0, \quad n \in \mathbb{Z}(1, T), \\
v_{0}(0)=v_{0}(T+1)=0 .
\end{gathered}
$$

Hence, the eigenvalue problem

$$
\begin{aligned}
\Delta^{2} v(n-1)+\lambda V^{\prime \prime}\left(u^{+}(n)\right) v(n) & =0, \quad n \in \mathbb{Z}(1, T), \\
v(0)=v(T+1) & =0
\end{aligned}
$$

has an eigenvalue $\lambda=1$. Condition $V^{\prime \prime}(t)>0$ implies that 1 must be a simple eigenvalue; see [1]. So, $\operatorname{dim} \operatorname{ker}\left(J^{\prime \prime}\left(u^{+}\right)\right)=1$. Since $E$ is finite dimensional, the Morse index of $u^{+}$must be finite and $J^{\prime \prime}\left(u^{+}\right)$must be a Fredholm operator. By Proposition 2.8, $C_{q}\left(J, u^{+}\right) \cong \delta_{q, 1} \mathbb{F}$. Finally, choose the neighborhood $U^{+}$of $u^{+}$such that $u>0$ for all $u \in U^{+}$, then

$$
C_{q}\left(J, u^{+}\right) \cong C_{q}\left(J^{+}, u^{+}\right) \cong \delta_{q, 1} \mathbb{F} \text {. }
$$

The proof is complete.

Lemma 3.4. $B y V^{\prime \prime}(0)<\lambda_{1}$, one has

$$
C_{q}(J, 0) \cong C_{q}\left(J^{+}, 0\right) \cong \delta_{q, 0} \mathbb{F} \text {. }
$$

Proof. By assumption, we have $J^{\prime+}(0)=J^{\prime}(0)=0$ and for all $u \in E \backslash\{0\}$,

$$
\left\langle J^{\prime \prime+}(0) u, u\right\rangle=\left\langle J^{\prime \prime}(0) u, u\right\rangle=\|u\|^{2}-V^{\prime \prime}(0) \sum_{n=1}^{T}|u(n)|^{2} \geq\left(1-\frac{V^{\prime \prime}(0)}{\lambda_{1}}\right)\|u\|^{2}>0,
$$

which implies that 0 is a local minimizer of both $J^{+}$and $J$. Hence, (3.21) holds.

Remark 3.5. Under the conditions of Theorem 1.3, we have

$$
C_{q}(J, \infty) \cong \delta_{q, k} \mathbb{F}
$$


We will use Propositions 2.4 and 2.5 to prove (3.23). Very similar to the proof of Lemma 3.2, we can prove that $J$ satisfies the (PS) condition. And it is easy to prove that $J$ satisfies (2.11). In fact, let

$$
X=\operatorname{span}\left\{\phi_{1}, \phi_{2}, \ldots, \phi_{k}\right\}, \quad Y=\operatorname{span}\left\{\phi_{k+1}, \ldots, \phi_{T}\right\} .
$$

By $V^{\prime \prime}(t) \leq \gamma<\lambda_{k+1}$, for all $x \in X$ and $y, y_{1} \in Y$, we have

$$
\begin{aligned}
\left\langle J^{\prime}(x+y)-J^{\prime}\left(x+y_{1}\right), y-y_{1}\right\rangle & =\left\|y-y_{1}\right\|^{2}-\sum_{n=1}^{T} V^{\prime \prime}(\xi)\left[y(n)-y_{1}(n)\right]^{2} \\
& \geq\left\|y-y_{1}\right\|^{2}-\gamma \sum_{n=1}^{T}\left[y(n)-y_{1}(n)\right]^{2} \\
& \geq\left\|y-y_{1}\right\|^{2}-\frac{\gamma}{\lambda_{k+1}}\left\|y-y_{1}\right\|^{2} .
\end{aligned}
$$

Hence, if we set $m=1-\gamma / \lambda_{k+1}$, then (2.11) holds.

Now, noticing that $V^{\prime \prime}(\infty) \in\left(\lambda_{k}, \lambda_{k+1}\right)$ implies that there exist $\bar{\gamma}>\lambda_{k}, \bar{\gamma}_{1}<\lambda_{k+1}$ and $b \in \mathbb{R}$ such that

$$
\frac{\bar{\gamma}}{2} t^{2}+b \leq V(t) \leq \frac{\bar{\gamma}_{1}}{2} t^{2}+b, \quad \text { for } t \in \mathbb{R}
$$

Hence, we have

$$
\begin{aligned}
& J(u) \longrightarrow-\infty, \quad \text { as } u \in X,\|u\| \longrightarrow \infty \\
& J(u) \longrightarrow+\infty, \quad \text { as } u \in Y,\|u\| \longrightarrow \infty .
\end{aligned}
$$

Then, (3.23) is proved by Propositions 2.4 and 2.5.

Remark 3.6. Following the proof of Theorem 3.1 in [17], (3.23) implies that there must exist a critical point $u_{0} \neq 0$ of $J$ satisfying

$$
C_{q}\left(J, u_{0}\right) \cong \delta_{q, k} \mathbb{F}
$$

It is known that the critical groups are useful in distinguishing critical points. So far, we have obtained four critical points $0, u^{+}, u^{-}$, and $u_{0}$ together with their characterization of critical groups. Assume that $0, u^{+}, u^{-}$, and $u_{0}$ are the only critical points of $J$. Then, the Morse inequality (2.10) becomes

$$
(-1)^{0}+(-1)^{1} \times 2+(-1)^{k}=(-1)^{k} .
$$

This is impossible. Thus, $J$ must have at least one more critical point $u_{1}$. Hence, (1.1) has at least five solutions. However, it seems difficult to obtain the sign property of $u_{0}$ and $u_{1}$. 
To obtain more refined results, we seek the third nontrivial solution $u_{0}$ to (1.1) by LyapunovSchmidt reduction method and then its characterization of the local degree results in one or two more nontrivial solutions to (1.1) together with their sign property.

Remark 3.7. The condition $k \geq 2$ in Theorem 1.3 is necessary to obtain three or more nontrivial solutions to (1.1). In fact, if $k=1$, then we have

$$
C_{q}\left(J, u_{0}\right) \cong C_{q}\left(J, u^{+}\right) \cong C_{q}\left(J, u^{-}\right) \cong \delta_{q, 1} \mathbb{F}
$$

Hence, $u_{0}$ may coincide with $u^{+}$or $u^{-}$which becomes an obstacle to seek other critical points by using Morse inequality. If $k=0$, then

$$
C_{q}\left(J, u_{0}\right) \cong C_{q}(J, 0) \cong \delta_{q, 0} \mathbb{F} \text {. }
$$

Hence, one cannot exclude the possibility of $u_{0}=0$.

To compute the degree of the set of positive (or negative) solutions to (1.1), we need the following lemma.

Lemma 3.8. There exists $\rho>0$ large enough, such that

$$
d\left(J^{\prime+}, B_{\rho}, 0\right)=d\left(J^{\prime-}, B_{\rho}, 0\right)=0
$$

Proof. We only prove the case of $J^{+}$. For any $\gamma_{1}>\lambda_{1}$, define $p\left(\gamma_{1}\right):=p$ as

$$
p(t)= \begin{cases}r_{1} t, & t \geq 0 \\ V^{\prime \prime}(0) t, & t<0\end{cases}
$$

Let $P(t)=\int_{0}^{t} p(s) d s$. The functional $Q: E \rightarrow \mathbb{R}$ is defined as

$$
Q(u)=\frac{1}{2}\|u\|^{2}-\sum_{n=1}^{T} P(u(n)) .
$$

It is obvious that $Q$ is of class $C^{1}$ and its critical points are precisely solutions to

$$
\begin{gathered}
\Delta^{2} u(n-1)+p(u(n))=0, \quad n \in \mathbb{Z}(1, T) \\
u(0)=0=u(T+1)
\end{gathered}
$$

Since $V^{\prime \prime}(0)<\lambda_{1}$, we see that if $u \neq 0$ is a solution to (3.36), then $u$ is positive. Because this contradicts $\gamma>\lambda_{1}$, we conclude that $u \equiv 0$ is the only critical point of $Q$. 
We claim that if $B$ is a ball in $E$ containing zero, then $d\left(Q^{\prime}, B, 0\right)=0$. In fact, since $\gamma_{1}>\lambda_{1}>V^{\prime \prime}(0)$ and $h(t):=p(t)-\lambda_{1} t>0$ for $t \neq 0$. Hence, for $u \in \partial B$, we have

$$
\begin{aligned}
\left\langle Q^{\prime}(u), \phi_{1}\right\rangle & =\sum_{n=0}^{T}\left(\Delta u(n), \Delta \phi_{1}(n)\right)-\sum_{n=1}^{T} \lambda_{1}\left(u(n), \phi_{1}(n)\right)-\sum_{n=1}^{T}\left(h(u(n)), \phi_{1}(n)\right) \\
& =-\sum_{n=1}^{T}\left(\Delta^{2} \phi_{1}(n-1)+\lambda_{1} \phi_{1}(n), u(n)\right)-\sum_{n=1}^{T}\left(h(u(n)), \phi_{1}(n)\right) \\
& =-\sum_{n=1}^{T}\left(h(u(n)), \phi_{1}(n)\right)<0,
\end{aligned}
$$

where we have used the fact that $\phi_{1}$ is positive on $\mathbb{Z}(1, T)$. Then, for each $s \in[0,1]$ and $u \in E \cap \partial B$, we have

$$
\left\langle s Q^{\prime}(u)+(1-s)\left(-\phi_{1}\right), \phi_{1}\right\rangle<0
$$

Hence, by invariance under homotopy of Brouwer degree, we have

$$
d\left(Q^{\prime}, B, 0\right)=d(K, B, 0)=0,
$$

where $K(u)=-\phi_{1}$.

Now, let $\gamma_{1}=V^{\prime \prime}(\infty)$. We claim that for $\rho>0$ large enough and for all $s \in[0,1]$, the function $s J^{\prime+}+(1-s) Q^{\prime}$ has no zero on $\partial B_{\rho}$.

In fact, we have proved that for all $\rho>0$ and for all $u \in \partial B_{\rho}$, we have

$$
\left\langle Q^{\prime}(u), \phi_{1}\right\rangle<0 \text {. }
$$

On the other hand, by the definition of $V^{\prime \prime}(\infty)$, for all $\epsilon>0$, there exists $\rho>0$ large enough such that $V^{\prime \prime}(\infty)-\epsilon<V^{\prime}(t) / t<V^{\prime \prime}(\infty)+\epsilon$ for $|t| \geq \rho$. Since $V^{\prime \prime}(\infty) \in\left(\lambda_{k}, \lambda_{k+1}\right)$, for $t \geq \rho$, take $\epsilon_{1}=\left(V^{\prime \prime}(\infty)-\lambda_{k}\right) / 2$, then

$$
\frac{V^{\prime}(t)}{t}>\frac{V^{\prime \prime}(\infty)+\lambda_{k}}{2}>\lambda_{k}
$$

For $t \leq-\rho$, take $\epsilon_{2}=\left(\lambda_{k+1}-V^{\prime \prime}(\infty)\right) / 2$, then

$$
\frac{V^{\prime}(t)}{t}<\frac{V^{\prime \prime}(\infty)+\lambda_{k+1}}{2}<\lambda_{k+1}
$$

Hence, if we take $\epsilon=\min \left\{\epsilon_{1}, \epsilon_{2}\right\}$, then for $t \geq \rho$, we have $V^{\prime}(t)>\lambda_{k} t>\lambda_{1} t$, and for $t \leq-\rho$, we have $V^{\prime}(t)>\lambda_{k+1} t$. So, if we let

$$
q(t):=V^{\prime+}(t)-\lambda_{1} t= \begin{cases}V^{\prime}(t)-\lambda_{1} t, & t \geq 0 \\ V^{\prime \prime}(0) t-\lambda_{1} t, & t<0\end{cases}
$$


then $q(t)>0$ for all $|t| \geq \rho$. And for all $u \in \partial B_{\rho}$, we have

$$
\begin{aligned}
\left\langle J^{\prime+}(u), \phi_{1}\right\rangle & =\sum_{n=0}^{T}\left(\Delta u(n), \Delta \phi_{1}(n)\right)-\sum_{n=1}^{T}\left(V^{\prime+}(u(n)), \phi_{1}(n)\right) \\
& =-\sum_{n=1}^{T}\left(\Delta^{2} \phi_{1}(n-1), u(n)\right)-\sum_{n=1}^{T}\left(V^{\prime+}(u(n)), \phi_{1}(n)\right) \\
& =\sum_{n=1}^{T}\left(\lambda_{1} \phi_{1}(n), u(n)\right)-\sum_{n=1}^{T}\left(V^{\prime+}(u(n)), \phi_{1}(n)\right) \\
& =-\sum_{n=1}^{T}\left(q(u(n)), \phi_{1}(n)\right)<0 .
\end{aligned}
$$

So far, we have proved that for $\rho>0$ large enough, $s J^{\prime+}+(1-s) Q^{\prime}$ has no zero point on $\partial B_{\rho}$ for each $s \in[0,1]$. Hence, by invariance under homotopy of Brouwer degree, we obtain

$$
d\left(J^{\prime+}, B_{\rho}, 0\right)=d\left(Q^{\prime}, B_{\rho}, 0\right)=0 .
$$

This completes the proof.

Remark 3.9. By Theorem 2.9 and the above results, we have the following characterization of degree of critical points.

(i) If $U^{+}\left(U^{-}\right)$is a neighborhood of $u^{+}\left(u^{-}\right)$containing no other critical points, then

$$
\begin{aligned}
& d\left(J^{\prime+}, U^{+}, 0\right)=d\left(J^{\prime}, U^{+}, 0\right)=-1, \\
& d\left(J^{\prime+}, U^{-}, 0\right)=d\left(J^{\prime}, U^{-}, 0\right)=-1 .
\end{aligned}
$$

(ii) Assume that $B$ is a ball centered at zero containing on other critical points, then

$$
d\left(J^{\prime+}, B, 0\right)=1=d\left(J^{\prime}, B, 0\right) .
$$

Hence, if $\Sigma$ is a bounded region containing the positive critical points and no other critical points, then by (3.33) we have

$$
\begin{aligned}
d\left(J^{\prime}, \Sigma, 0\right) & =d\left(J^{\prime+}, \Sigma, 0\right)=d\left(J^{\prime+}, B_{\rho}-\bar{B}, 0\right) \\
& =d\left(J^{\prime+}, B_{\rho}, 0\right)-d\left(J^{\prime+}, B, 0\right)=-1 .
\end{aligned}
$$

Similarly, we see that if $\Sigma_{1}$ is a bounded region containing the negative critical points and no other critical points, then

$$
d\left(J^{\prime}, \Sigma_{1}, 0\right)=-1
$$


Now, we can give the proof of Theorem 1.3.

Proof of Theorem 1.3. The functional $J$ satisfies (2.18) in Lemma 2.10 due to the fact that $J$ satisfies (2.11). Hence, by Lemma 2.10 , there exists $\psi: X \rightarrow \mathbb{R}$ such that

$$
J(x+\psi(x))=\min _{y \in Y} J(x+y)
$$

Moreover, $\psi(x)$ is the unique member of $Y$ such that

$$
\left\langle J^{\prime}(x+\psi(x)), y\right\rangle=0, \quad \forall y \in Y .
$$

The function $\bar{J}: X \rightarrow \mathbb{R}$ defined by $\bar{J}(x)=J(x+\psi(x))$ is of class $C^{1}$. Because $\bar{J}(x) \leq J(x),(3.27)$ implies that $\bar{J}(x) \rightarrow-\infty$ as $\|x\| \rightarrow \infty$. Since $\operatorname{dim} X<\infty$, there must exist $x_{0} \in X$ such that $\bar{J}\left(x_{0}\right)=\max _{x \in X} J(x+\psi(x))$. Take $u_{0}=x_{0}+\psi\left(x_{0}\right)$, then $J^{\prime}\left(u_{0}\right)=0$ by (iii) of Lemma 2.10. If $V$ is a neighborhood of $u_{0}$ containing no other critical points of $J$, taking $W=\{x \in X: x+\psi(x) \in$ $V\}$, then $d\left(\bar{J}^{\prime}, W, 0\right)=(-1)^{k}$. Then, by part (iv) of Lemma 2.10, we have

$$
d\left(J^{\prime}, V, 0\right)=(-1)^{k}
$$

\section{Suppose that $k$ Is Even}

Let $R_{1}$ be large enough so that if $\bar{J}^{\prime}(x)=0$, then $\|x\|<R_{1}$. Because $\operatorname{dim} X<\infty$ and $\psi(x)$ is of class $C^{1}$, there exists $R_{2}>0$ such that $\|\psi(x)\|<R_{2}$ for $\|x\|<R_{1}$. Because $-\bar{J}$ is coercive, $d\left(\bar{J}^{\prime}, B_{R_{1}}, 0\right)=(-1)^{k}$. Hence, if we set $C=\left\{x+y:\|x\|<R_{1},\|y\|<R_{2}\right\}$, then by (iv) of Lemma 2.10, we have

$$
d\left(J^{\prime}, C, 0\right)=d\left(\bar{J}^{\prime}, B_{R_{1}}, 0\right)=(-1)^{k}=1
$$

Suppose that $\mathcal{K}=\left\{u \in E \mid J^{\prime}(u)=0\right\}$ is finite. Let $S_{1}, S_{2}$, and $S_{3}$ be disjoint open bounded regions in $E$ such that $\bar{S}_{1} \cap \mathcal{K}=\{0\}, \bar{S}_{2} \cap \mathcal{K}$ is the set of positive critical points of $J$, and $\bar{S}_{3} \cap \mathcal{K}$ is the set of negative critical points of $J$. So far, we have proved that

$$
d\left(J^{\prime}, S_{1}, 0\right)=1, \quad d\left(J^{\prime}, S_{2}, 0\right)=d\left(J^{\prime}, S_{3}, 0\right)=-1 .
$$

(i) If $u_{0}=x_{0}+\psi\left(x_{0}\right) \notin S_{2} \cup S_{3}$, then $u_{0}$ is sign changing. Let $S_{4}$ denote an open bounded region disjoint from $\overline{S_{1} \cup S_{2} \cup S_{3}}$ such that $\bar{S}_{4} \cap \mathcal{K}=\left\{u_{0}\right\}$. By the excision property of Brouwer degree, we have

$$
\begin{aligned}
-1= & d\left(J^{\prime}, C, 0\right)=d\left(J^{\prime}, S_{1}, 0\right)+d\left(J^{\prime}, S_{2}, 0\right)+d\left(J^{\prime}, S_{3}, 0\right)+d\left(J^{\prime}, S_{4}, 0\right) \\
& +d\left(J^{\prime}, C-\overline{S_{1} \cup S_{2} \cup S_{3} \cup S_{4}}, 0\right) \\
= & 1-1-1+1+d\left(J^{\prime}, C-\overline{S_{1} \cup S_{2} \cup S_{3} \cup S_{4}}, 0\right),
\end{aligned}
$$


Thus, by Kronecker existence property of Brouwer degree, we see that there must exist $u_{1} \in$ $C-\overline{S_{1} \cup S_{2} \cup S_{3} \cup S_{4}}$ such that $J^{\prime}\left(u_{1}\right)=0$, which proves that (1.1) has at least five solutions. In this case, both $u_{0}$ and $u_{1}$ change sign.

(ii) Suppose now that $u_{0} \in S_{2} \cup S_{3}$. Without loss of generality, we may assume that $u_{0} \in S_{2}$. Let $S_{4,2}$ be a neighborhood of $u_{0}$ such that $\bar{S}_{4,2} \cap \mathcal{K}=\left\{u_{0}\right\}$. By Lemma 3.3, there exists a critical point of mountain pass type $u^{+} \in S_{2}$ such that if $S_{5}$ is a neighborhood of $u^{+}$such that $S_{5} \cap \mathcal{K}=\left\{u^{+}\right\}$, then $d\left(J^{\prime}, S_{5}, 0\right)=-1$. Thus,

$$
\begin{aligned}
-1 & =d\left(J^{\prime}, S_{2}, 0\right)=d\left(J^{\prime}, S_{4,2}, 0\right)+d\left(J^{\prime}, S_{5}, 0\right)+d\left(J^{\prime}, S_{2}-\overline{S_{4,2} \cup S_{5}}, 0\right) \\
& =1-1+d\left(J^{\prime}, S_{2}-\overline{S_{4,2} \cup S_{5}}, 0\right) .
\end{aligned}
$$

Thus, by Kronecker existence property of Brouwer degree, there exists $u_{1} \in S_{2}-\overline{S_{4,2} \cup S_{5}}$ such that $J^{\prime}\left(u_{1}\right)=0$. Finally,

$$
\begin{aligned}
1=d\left(J^{\prime}, C, 0\right)= & d\left(J^{\prime}, S_{1}, 0\right)+d\left(J^{\prime}, S_{2}, 0\right)+d\left(J^{\prime}, S_{3}, 0\right) \\
& +d\left(J^{\prime}, C-\overline{S_{1} \cup S_{2} \cup S_{3}}, 0\right) \\
= & 1-1-1+d\left(J^{\prime}, C-\overline{S_{1} \cup S_{2} \cup S_{3}}, 0\right) .
\end{aligned}
$$

Thus, there must exist $u_{2} \in C-\overline{S_{1} \cup S_{2} \cup S_{3}}$ such that $J^{\prime}\left(u_{2}\right)=0$. Thus, the set $\left\{u^{+}, u_{0}, u_{1}, u_{2}\right\}$ together with a critical point $u^{-}$of $J$ in $S_{3}$ shows that (1.1) has five nontrivial solutions. Since $u_{2} \notin S_{2} \cup S_{3}$ and $u^{+}, u_{0}, u_{1} \in S_{2}, u_{2}$ is a sign-changing solution, and $u^{+}, u_{0}$, and $u_{1}$ have the same sign. This completes the proof of Theorem 1.3, when $k$ is even.

\section{Suppose that $k$ Is Odd}

(iii) Let $S_{1}, S_{2}$, and $S_{3}$ be as above. If $u_{0} \notin S_{2} \cup S_{3}$, the proof follows very closely that of the case (i).

(iv) Suppose that $u_{0}=x_{0}+\psi\left(x_{0}\right) \in S_{2} \cup S_{3}$, hence $u_{0} \in S_{2}$. Because $u_{0}>0$, there exists $\epsilon>0$ such that $x+\psi(x)>0$ if $\left\|x-x_{0}\right\|<\epsilon$. So, if $x \in\left\{x:\left\|x-x_{0}\right\|<\epsilon\right\}$, then $\bar{J}=\bar{J}^{+}$and $x_{0}$ is a local maximum of $\bar{J}^{+}$. Since we are assuming (1.1) to have only finitely many solutions, $x_{0}$ is a strictly local maximum of $\bar{J}^{+}$. Let $\delta>0$ be such that $\bar{J}^{+}(x)<\bar{J}^{+}\left(x_{0}\right)$ if $0<\left\|x-x_{0}\right\|<\delta$. Since $k \geq 2,\left\{x: 0<\left\|x-x_{0}\right\|<\epsilon\right\}$ is path connected. Thus, $x_{0}$ is not a critical point of mountain pass type. By Lemma 3.3, $J^{+}$has a critical point of mountain pass type $u^{+}=x^{+}+\psi\left(x^{+}\right)$. By (v) of Lemma 2.10, $x_{0} \neq x^{+}$, and hence $u_{0} \neq u^{+}$. Let $V_{0}, V_{1}$ be neighborhoods of $u_{0}$ and $u^{+}$, respectively, such that $\bar{V}_{0} \cap \mathcal{K}=\left\{u_{0}\right\}$ and $\bar{V}_{1} \cap \mathcal{K}=\left\{u^{+}\right\}$. Thus,

$$
\begin{aligned}
-1 & =d\left(J^{\prime+}, S_{2}, 0\right)=d\left(J^{\prime+}, V_{0}, 0\right)+d\left(J^{\prime+}, V_{1}, 0\right)+d\left(J^{\prime+}, S_{2}-\overline{V_{0} \cup V_{1}}, 0\right) \\
& =-2+d\left(J^{\prime+}, S_{2}-\overline{V_{0} \cup V_{1}}, 0\right) .
\end{aligned}
$$


Thus, by Kronecker existence property of Brouwer degree, there exists a third positive solution $u_{1} \in S_{2}-\overline{V_{0} \cup V_{1}}$. So far, we have proved that (1.1) has at least four nontrivial solutions $\left\{u^{-}, u^{+}, u_{0}, u_{1}\right\}$ and that $u^{+}, u_{0}, u_{1} \in S_{2}$ have the same sign. This proves Theorem 1.3.

\section{Acknowledgments}

Project supported by National Natural Science Foundation of China (no. 11026059) and Foundation for Distinguished Young Talents in Higher Education of Guangdong, China (no. LYM09105).

\section{References}

[1] R. P. Agarwal, Difference Equations and Inequalities: Theory, Methods, and Applications, vol. 228 of Monographs and Textbooks in Pure and Applied Mathematics, Marcel Dekker, New York, NY, USA, 2nd edition, 2000.

[2] A. N. Sharkovsky, Y. L. Mařstrenko, and E. Y. Romanenko, Difference Equations and Their Applications, vol. 250 of Mathematics and Its Applications, Kluwer Academic, Dordrecht, The Netherlands, 1993.

[3] R. M. May, "Simple mathematical models with very complicated dynamics," Nature, vol. 261, pp. 459-466, 1976.

[4] R. P. Agarwal, K. Perera, and D. O’Regan, "Multiple positive solutions of singular and nonsingular discrete problems via variational methods," Nonlinear Analysis. Theory, Methods \& Applications, vol. 58, no. 1-2, pp. 69-73, 2004.

[5] B. Zheng and Q. Zhang, "Existence and multiplicity of solutions of second-order difference boundary value problems," Acta Applicandae Mathematicae, vol. 110, no. 1, pp. 131-152, 2010.

[6] X. Cai and J. Yu, "Existence theorems for second-order discrete boundary value problems," Journal of Mathematical Analysis and Applications, vol. 320, no. 2, pp. 649-661, 2006.

[7] L. Jiang and Z. Zhou, "Existence of nontrivial solutions for discrete nonlinear two point boundary value problems," Applied Mathematics and Computation, vol. 180, no. 1, pp. 318-329, 2006.

[8] H. Liang and P. Weng, "Existence and multiple solutions for a second-order difference boundary value problem via critical point theory," Journal of Mathematical Analysis and Applications, vol. 326, no. 1, pp. 511-520, 2007.

[9] M. Aprahamian, D. Souroujon, and S. Tersian, "Decreasing and fast solutions for a second-order difference equation related to Fisher-Kolmogorov's equation," Journal of Mathematical Analysis and Applications, vol. 363, no. 1, pp. 97-110, 2010.

[10] J. Mawhin and M. Willem, Critical Point Theory and Hamiltonian Systems, vol. 74 of Applied Mathematical Sciences, Springer, New York, NY, USA, 1989.

[11] K.-C. Chang, Infinite-Dimensional Morse Theory and Multiple Solution Problems, Birkhäuser, Boston, Mass, USA, 1993.

[12] W. Takahashi, Nonlinear Functional Analysis, Yokohama Publishers, Yokohama, Japan, 2000.

[13] G. Cerami, "An existence criterion for the critical points on unbounded manifolds," Istituto Lombardo. Accademia di Scienze e Lettere. Rendiconti A, vol. 112, no. 2, pp. 332-336, 1978 (Italian).

[14] P. Bartolo, V. Benci, and D. Fortunato, "Abstract critical point theorems and applications to some nonlinear problems with "strong" resonance at infinity," Nonlinear Analysis. Theory, Methods $\mathcal{E}$ Applications, vol. 7, no. 9, pp. 981-1012, 1983.

[15] K. C. Chang, "Solutions of asymptotically linear operator equations via Morse theory," Communications on Pure and Applied Mathematics, vol. 34, no. 5, pp. 693-712, 1981.

[16] T. Bartsch and S. J. Li, "Critical point theory for asymptotically quadratic functionals and applications to problems with resonance," Nonlinear Analysis. Theory, Methods E Applications, vol. 28, no. 3, pp. 419 441, 1997.

[17] S. Liu and S. Li, "Critical groups at infinity, saddle point reduction and elliptic resonant problems," Communications in Contemporary Mathematics, vol. 5, no. 5, pp. 761-773, 2003.

[18] A. Castro and J. Cossio, "Multiple solutions for a nonlinear Dirichlet problem," SIAM Journal on Mathematical Analysis, vol. 25, no. 6, pp. 1554-1561, 1994. 